\title{
Bosentan Improves Skin Perfusion of Hands in Patients with Systemic Sclerosis with Pulmonary Arterial Hypertension
}

\author{
EDOARDO ROSATO, ILENIA MOLINARO, FEDERICA BORGHESE, CARMELINA ROSSI, SIMONETTA PISARRI, \\ and FELICE SALSANO
}

ABSTRACT. Objective. Our aim was to investigate effects of bosentan on hand perfusion in patients with systemic sclerosis (SSc) with pulmonary arterial hypertension (PAH), using laser Doppler perfusion imaging (LDPI).

Methods. We enrolled 30 SSc patients with PAH, 30 SSc patients without PAH, and 30 healthy controls. In SSc patients and healthy controls at baseline, skin blood flow of the dorsum of the hands was determined with a Lisca laser Doppler perfusion imager. The dorsal surface of the hands was divided into 3 regions of interest (ROI). ROI 1 included 3 fingers of the hand from the second to the fourth distally to the proximal interphalangeal finger joint. ROI 2 included the area between the proximal interphalangeal and the metacarpophalangeal joint. ROI 3 included only the dorsal surface of the hand without the fingers. LDPI was repeated in SSc patients and controls after 4, 8, and 16 weeks of treatment. In SSc patients, nailfold videocapillaroscopy and Raynaud Condition Score (RCS) were performed at baseline and at 4, 8, and 16 weeks.

Results. SSc patients with PAH enrolled in the study received treatment with bosentan as standard care for PAH. In these patients with PAH, after 8 and 16 weeks of treatment, bosentan improved minimum, mean, and maximum perfusion and the perfusion proximal-distal gradient. Bosentan seems to be most effective in patients with the early and active capillaroscopic pattern than in patients with the late pattern. Bosentan improved skin blood flow principally in the ROI 1 compared to the ROI 2 and ROI 3. Bosentan restored the perfusion proximal-distal gradient in 57\% of SSc patients with the early capillaroscopic pattern. No significant differences from baseline were observed in the RCS in SSc patients with PAH.

Conclusion. Bosentan improved skin perfusion in SSc patients with PAH, although it did not ameliorate symptoms of Raynaud's phenomenon. Skin blood perfusion increased in SSc patients with $\mathrm{PAH}$, particularly in the skin region distal to the proximal interphalangeal joint, and in patients with the early/active capillaroscopic pattern. Double-blind randomized clinical trials are needed to evaluate the effects of bosentan on skin perfusion of SSc patients without PAH and with active digital ulcers. (First Release September 1 2010; J Rheumatol 2010;37:2531-9; doi:10.3899/jrheum.100358)

Key Indexing Terms:

BOSENTAN

RAYNAUD'S PHENOMENON

\section{LASER DOPPLER PERFUSION IMAGING SYSTEMIC SCLEROSIS SKIN BLOOD FLOW}

Systemic sclerosis ( $\mathrm{SSc}$ ) is a clinically heterogeneous disorder of connective tissue characterized by immune activation, microvascular injury, and fibrosis ${ }^{1}$. Independently of the classification of the disease, SSc is typically associated with Raynaud's phenomenon (RP) ${ }^{2}$. RP secondary to SSc is characterized by microvascular damage and high plasma

From the Department of Clinical Medicine, Clinical Immunology Unit - Scleroderma Center, Sapienza University of Rome, Rome, Italy. E. Rosato, MD, PhD; I. Molinaro, Medical Student; F. Borghese, MD; C. Rossi, MD; S. Pisarri, MD, Professor of Internal Medicine; F. Salsano, $M D$, Professor of Internal Medicine, Director, Clinical Immunology Unit. Address correspondence to Prof. F. Salsano, Department of Clinical Medicine, Sapienza University of Rome, Viale dell'Università 37, 00185 Rome, Italy.E-mail: felice.salsano@uniroma1.it

Accepted for publication July 12, 2010. levels of adrenomedullin and endothelin-1 (ET-1). Excessive vasoconstrictive response leads to pallor and cyanosis of distal extremities, particularly of the digits, with complications including digital ulceration and infarction ${ }^{3,4,5}$.

In recent years, ET-1 has been strongly implicated in the pathogenesis of SSc and pulmonary arterial hypertension $(\mathrm{PAH})$. As well as being a potent vasocontrictor, ET-1 affects vascular remodeling, and it is therefore possible that bosentan, by blocking ET-1, might favorably influence the structural vascular abnormality that is known to play a major role in SSc-related digital vascular disease. It has recently been shown that the ET-1 receptor antagonist bosentan improves PAH and digital ulceration secondary to $\mathrm{SSc}^{6,7}$.

Several noninvasive techniques for evaluating perfusion Personal non-commercial use only. The Journal of Rheumatology Copyright @ ${ }^{2010 . ~ A l l ~ r i g h t s ~ r e s e r v e d . ~}$ 
of the hand and wrist arteries have been brought into clinical practice 8,9 . Recently, Rosato, et al showed that laser Doppler perfusion imaging (LDPI) is a noninvasive technique for evaluating skin blood flow in patients with primary RP and $\mathrm{SSc}^{9}$.

The aim of our study was to investigate skin blood perfusion of dorsal hands by LDPI at baseline and during treatment with bosentan in SSc patients with PAH.

\section{MATERIALS AND METHODS}

Subjects. Sixty patients with SSc (52 female, 8 male; mean age $55.5 \pm 12.5$ yrs) followed in our center were enrolled for this study. All SSc patients fulfilled the American College of Rheumatology criteria for $\mathrm{SSc}^{10}$ and they were subsequently divided into the limited (lcSSc) and diffuse ( $\mathrm{dcSSc}$ ) cutaneous SSc groups, according to LeRoy, et $a l^{1}$.

Thirty patients with SSc had PAH (25 female, 5 male; mean age $58 \pm$ 12 yrs), diagnosed according to American College of Chest Physicians (ACCP) guidelines ${ }^{11}$. As all patients with SSc fulfilled the ACCP criteria for PAH, bosentan therapy was started and was continued during the time of the study. The other $30 \mathrm{SSc}$ patients without pulmonary hypertension (27 female, 3 male; mean age $53 \pm 13 \mathrm{yrs}$ ) and 30 healthy controls matched for sex and age ( 25 female, 5 male; mean age $56 \pm 11$ yrs) were also enrolled. Table 1 lists the main baseline epidemiological and clinical features of the patients.

Disease activity and disease severity in SSc patients were measured by the Valentini Disease Activity Index ${ }^{12}$ and the Medsger Disease Severity Scale $^{13}$, respectively.

Subjects' written consent was obtained according to the Declaration of Helsinki and the study was approved by the local ethics committee.

SSc patients with active digital ulcers were excluded from the study. No patient had received treatment with parenteral prostanoids or bosentan for at least 3 months prior to this study point. Patients in current therapy with prostacyclin analogs and phosphodiesterase 5 inhibitors, with previous therapy with endothelin receptor antagonists, use of other endothelin receptor antagonists, prior sympathectomy of the upper limb, or with a history of uncontrolled systemic hypertension, hyperlipidemia, cardiac failure, hepat- ic failure, diabetics, peripheral vascular diseases, coagulopathy, smokers, pregnant or breastfeeding women, or those unable to give written informed consent were excluded.

SSc patients with and without PAH underwent therapeutic treatment with calcium channel blockers (nifedipine $30 \mathrm{mg} /$ day). Mean duration of therapy was $12 \pm 11$ and $11 \pm 9$ years, respectively. Therapy was discontinued 48 hours before the examination according to our previous findings 9 . Study design. The investigation was designed as an open-label, single-center study and was conducted over the cold winter months to maximize the development of RP as well as to minimize the seasonal effects on RP. Patients with SSc were recruited in the winter of 2005, 2006, 2007, and 2008. In patients who started therapy with bosentan before 2008 the diagnosis of pulmonary hypertension had been made by echocardiography. We chose a tricuspid gradient of $40 \mathrm{~mm} \mathrm{Hg}$ [i.e., an estimated systolic pulmonary artery pressure (PAP) $\geq 40+10=50 \mathrm{~mm} \mathrm{Hg}$ ] to determine moderate to severe pulmonary hypertension. At the time of echocardiography examination all patients had dyspnea class III or IV, graded according to the New York Heart Association (NYHA). All patients underwent high-resolution computed tomography (HRCT) of the chest and pulmonary function testing (PFT) after echocardiography to examine for interstitial lung disease (ILD) associated with scleroderma. Severe ILD (defined by forced vital capacity $<60 \%$ of predicted) was an exclusion criterion for bosentan therapy. PAH was confirmed by right heart catheterization in 4 patients who started bosentan therapy after January 2008. In all patients with PAH carbon monoxide diffusing capacity (DLCO $\%$, mean $\pm \mathrm{SD}$ ) was $62.7 \pm 25.9 \%$.

SSc patients with PAH started therapy with bosentan orally, $62.5 \mathrm{mg}$ bid for 4 weeks, followed by the target dose of $125 \mathrm{mg}$ bid. SSc patients with PAH enrolled in the study received bosentan treatment as the standard care for PAH.

SSc patients without PAH also underwent echocardiography, HRCT and PFT. We chose a tricuspid regurgitation velocity $\leq 2.8 \mathrm{~m} / \mathrm{s}$ (PAP $\leq 36$ $\mathrm{mm} \mathrm{Hg}$ ) to exclude pulmonary hypertension (Table 1). Severe ILD (defined by forced vital capacity $<60 \%$ of predicted) was an exclusion criterion for enrollment in this study. In all patients without PAH, mean DLCO\% was $73.5 \pm 21 \%$ and NYHA class was I or II. During the study the healthy controls did not receive any medication, while SSc patients without PAH continued therapy with calcium channel blockers (Figure 1).

Table 1. SSc patients' epidemiological and clinical features.

\begin{tabular}{|c|c|c|}
\hline Feature & SSc Patients with PAH & SSc Patients without PAH \\
\hline No. female/male & $25 / 5$ & $27 / 3$ \\
\hline Age, mean $\pm \mathrm{SD}$, yrs & $55 \pm 13$ & $53 \pm 13$ \\
\hline $\mathrm{SSc}$ duration since diagnosis, mean \pm SD yrs & $10 \pm 9$ & $11 \pm 11$ \\
\hline Raynaud's phenomenon duration, mean \pm SD yrs & $16 \pm 10$ & $15 \pm 13$ \\
\hline Subset of dcSSc/lcSSc, n* & $14 / 16$ & $15 / 15$ \\
\hline History of digital ulcers, $\mathrm{n}$ & 13 & 14 \\
\hline Systolic pulmonary artery pressure, mean $\pm \mathrm{SD} \mathrm{mm} \mathrm{Hg}$ & $58.5 \pm 10.8$ & $23 \pm 7$ \\
\hline \multicolumn{3}{|l|}{ ANA pattern, $\mathrm{n}(\%)$} \\
\hline Speckled & $5(17)$ & $6(20)$ \\
\hline Speckled and nucleolar & $2(7)$ & $1(3)$ \\
\hline Nucleolar & $11(36)$ & $10(33)$ \\
\hline Centromere & $12(40)$ & $13(44)$ \\
\hline \multicolumn{3}{|l|}{ SSc-specific autoantibody, n (\%) } \\
\hline Anti-topo I & $10(33)$ & $12(40)$ \\
\hline ACA & $13(43)$ & $14(47)$ \\
\hline None & $7(24)$ & $4(13)$ \\
\hline Disease Activity Index ${ }^{12}$ & $2.4 \pm 1.8$ & $2.5 \pm 2.1$ \\
\hline Disease Severity Scale ${ }^{13}$ & $4.8 \pm 2.5$ & $5.1 \pm 3.1$ \\
\hline
\end{tabular}

* Subset according to LeRoy, et $a l^{1}$. PAH: pulmonary arterial hypertension; dcSSc: diffuse cutaneous SSc; lcSSc: limited cutaneous SSc; ANA: antinuclear antibodies; anti-topo I: antitopoisomerase I; ACA: anticentromere antibody. 


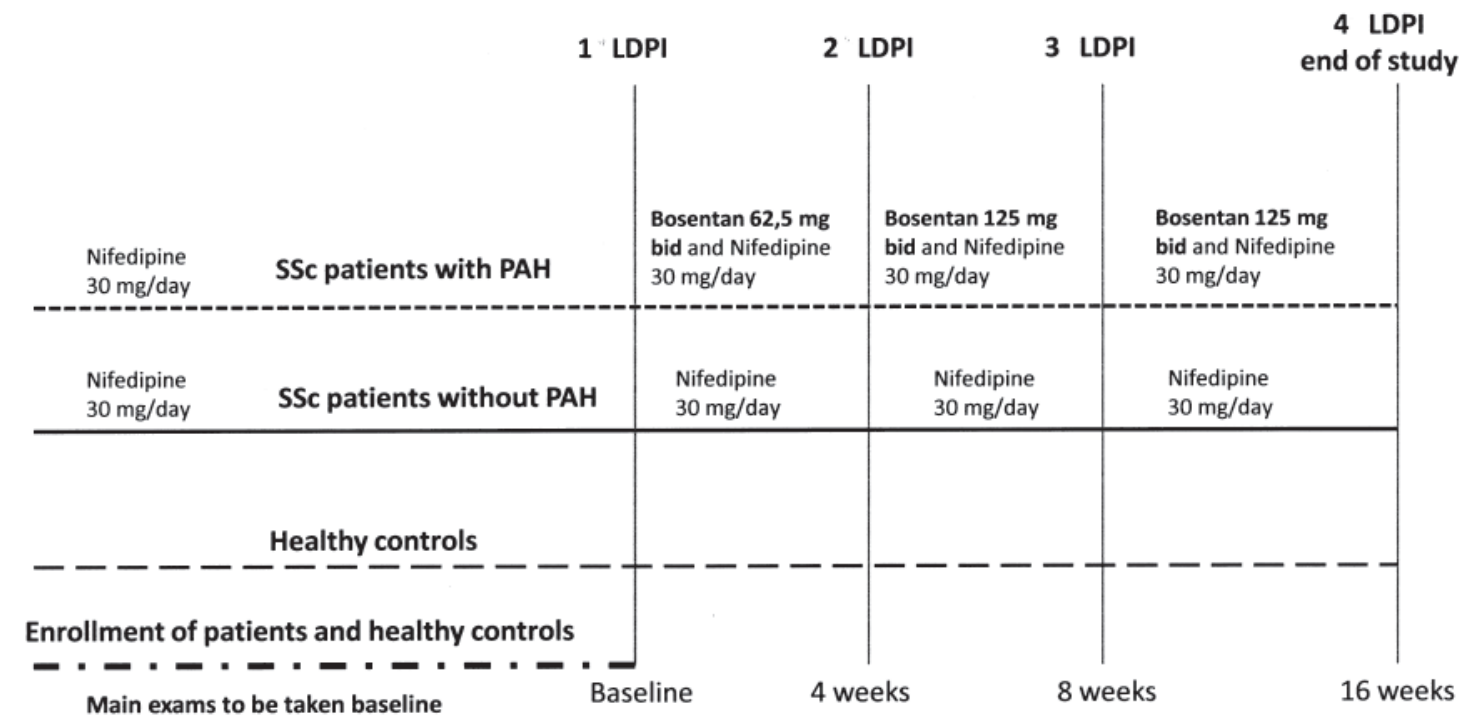

Figure 1. The design of the study. LDPI: laser Doppler perfusion imaging; PAH: pulmonary artery hypertension.

The primary outcome measure was the change in skin blood perfusion of the dorsum of the hands, measured by LDPI. All participants completed a set of outcome measurement instruments at the baseline pretreatment visit and after 4,8 , and 16 weeks, as described below.

The secondary outcomes were the changes in microvascular architecture, assessed by nailfold videocapillaroscopy (NVC), and in severity of RP attacks, measured by the Raynaud Condition Score (RCS).

In SSc patients with PAH, all LDPI measurements and NVC assessments were repeated at $4 \mathrm{~h}$ post-bosentan, except the RCS, which were not requested at $4 \mathrm{~h}$. The data were subjected to repeated-measures analysis variance. All recruited patients completed the study.

LDPI assessment. LDPI was performed in a quiet, air conditioned room $\left(24^{\circ} \mathrm{C} \pm 0.4\right)$ when subjects adjusted to the room temperature, after around 20 minutes. Patients and controls drank no coffee or alcoholic beverages for 2 days before the examination. A baseline scan of dorsal surfaces of both hands was performed in patients and controls. Skin blood flow of the dorsum of the hands was determined using a low-energy $670 \mathrm{~nm}$ Lisca laser Doppler perfusion imager (Perimed AB, Stockholm, Sweden). The scanner was placed perpendicularly $15 \mathrm{~cm}$ away from the hands. The laser sequentially scans $4096(64 \times 64$ pixels $)$ measurement points over the tissue under study, covering a maximal surface area of approximately $12 \mathrm{~cm} \times 12 \mathrm{~cm}$. Two-dimensional images of superficial tissue blood perfusion were acquired at the highest time and spatial resolution. All perfusion signals were combined to form a color-coded image using a scale ranging from dark blue (lowest value) to red (highest value). The term commonly used to describe blood flow measurements by the laser Doppler technique is "flux"; the flux is expressed in arbitrary perfusion units (pU), calculated using the first moment of the power spectral density. Each measured point has a numeric pU between 0.0 and $10.0 \mathrm{~V}$.

According to our previous study the dorsal surface of the hands was divided into 3 regions of interest: ROI 1, ROI 2, and ROI 3. ROI 1 included 3 fingers of the hand from second to fourth, distal to the proximal interphalangeal joint. ROI 2 included the area between the proximal interphalangeal and the metacarpophalangeal joint. ROI 3 included only the dorsal surface of the hand without the fingers.

The perfusion proximal-distal gradient and the perfusion distribution pattern were evaluated as defined in our previous study ${ }^{9}$. Skin blood flow was measured in only 3 fingers (index, middle, and ring). According to our previous study, the proximal-distal gradient is present when the difference of ROI 1 perfusion mean - ROI 2 perfusion mean is $>0.3 \mathrm{pU}$. For evaluation of the distribution pattern we used the color image aspect of ROI 2 and
ROI 3. ROI 1 was excluded in the definition of perfusion pattern because in patients with SSc the skin of this region may have digital ulcers, which appear in the LDPI as hyperperfused and hypoperfused areas. In addition, because of the small size of ROI 1, assessment of the distribution pattern is more difficult, even if it is possible. Instead, in ROI 2 and ROI 3 the presence of very small hypoperfused and hyperperfused areas is due to microvascular damage. In each color-coded image the homogeneous pattern was defined as a pattern showing structural uniformity of perfusion in ROI 2 and ROI 3, while a dyshomogeneous pattern was defined as a pattern showing the presence of very small hypoperfused and hyperperfused areas variously distributed in ROI 2 and ROI 3 . In addition, for each image, the instrument provided the following numeric values expressed in arbitrary $\mathrm{pU}$ : minimum perfusion, mean perfusion, maximum perfusion, with standard deviation (SD). The SD according to our previous study is an index of the presence of the proximal-distal gradient. The SD is very high in healthy controls for presence of the proximal-distal gradient, while SD is low in patients with SSc. The SD is different in 3 capillaroscopic patterns of SSc, as follows. In patients with the capillaroscopic active pattern, the $\mathrm{SD}$ was equal to that in healthy controls, because in this group hyperperfused and hypoperfused areas were present. In the group with the capillaroscopic late pattern, SD was very low because of marked reduction of the perfusion within a few hyperperfused areas. In the group with the capillaroscopic early pattern, SD was intermediate between the other 2 groups for initial sclerodermic microangiopathy in the absence of hypoperfused and hyperperfused areas.

In patients with SSc with sclerodactyly, LDPI was performed only when the maximal flexion of the fingers, evaluated as the point of maximum distance from the surface scan, was $<5 \mathrm{~mm}$. Indeed, in our experience, sclerodactyly with flexion of the fingers $<15 \mathrm{~mm}$ does not cause significant changes in the LDPI values ${ }^{9}$.

We acquired 2 LDPI images every 2 hours for each SSc patient and control to reduce variability and increase reproducibility. For the second LDPI measurement, patients and controls reattended for a further acclimatization of 20 minutes. The mean value was calculated for numerical measures, while the overlap between each color-coded image was assessed for evaluation of the perfusion distribution.

The operator who analyzed the LDPI results was blinded to patient diagnosis and capillaroscopy findings.

Nailfold videocapillaroscopy. The same operator, blinded to patient diagnosis, performed the NVC in each patient using an optical videocapillaroscope probe equipped with a 100-power contact lens and connected to 
image analysis software (Pinnacle Studio Version 8; Pinnacle Systems, Mountain View, CA, USA).

Each subject was inside the building for a minimum of 15 minutes before the nailfold was examined at a room temperature of $20-22^{\circ} \mathrm{C}$. The nailfold (distal row) of the second, third, fourth, and fifth finger was examined in each patient. The following capillaroscopic variables were considered according to previous observations: presence of enlarged and giant capillaries, hemorrhages, loss of capillaries, disorganization of the microvascular array, and capillary ramifications. According to Cutolo, et al, the capillaroscopic patterns were classified as early, active, and late ${ }^{14}$.

$R P$ attack diary. Every day for 2 weeks patients were instructed to record the total number and duration of each RP attack and the overall discomfort due to RP attacks, expressed by RCS, on a 0-10 ordinal scale (0: subject did not feel handicapped; 10: subject felt extremely handicapped by RP). The RCS incorporates the cumulative daily frequency, duration, severity, and effects of RP attacks ${ }^{15}$. Data from the RP attack diary were averaged for the 2 -week period preceding the baseline visit and over the 2 -week periods prior to the 4,8 , and 16 week post-treatment assessment visits.

Statistical analysis. SPSS version 15.0 (SPSS, Chicago, IL, USA) was used for the statistical analysis. Results were expressed as frequencies and percentages for binary and categorical variables and as mean \pm SD for continuous data. Comparative analyses were performed using chi-square or Fisher exact tests for categorical data. Paired and unpaired Student's t test was used for continuous data. All calculated $\mathrm{p}$ values were 2-tailed, and $\mathrm{p}$ values $<0.05$ were considered significant. The coefficient of skewness and coefficient of kurtosis were used to evaluate normal distribution of numerical variables. All continuous data were normally distributed.

\section{RESULTS}

Laser Doppler perfusion imaging. At baseline in the healthy controls the perfusion distribution pattern was homogeneous with the presence of a proximal-distal perfusion gradient. Conversely, in the patients with SSc the perfusion distribution pattern was dyshomogeneous and the proximal-distal gradient was absent (Table 2). In SSc patients the mean values of minimum, mean, and maximum perfusion and SD were significantly lower than those in healthy controls (Table 3). In the controls the ROI 1 perfusion was higher than that in ROI 2 and ROI 3. In controls the mean perfusion of ROI 1 was higher than in SSc patients and the distribution pattern of ROI 1 was homogeneous (Table 4). In con- trols ROI 2 and ROI 3 had homogeneous distribution, with higher mean perfusion than in patients with SSc.

In the $3 \mathrm{SSc}$ patient capillaroscopic groups (early, active, and late) the mean values of minimum, mean, and maximum perfusion and SD were different. In the group with the capillaroscopic active pattern the mean values of these 3 variables were high, while in the capillaroscopic-late group the values were very low. In the early capillaroscopic group the mean values of minimum, mean, and maximum perfusion and SD were intermediate between the other 2 groups (Table 5).

In $30 \mathrm{SSc}$ patients without $\mathrm{PAH}$ and healthy controls, no significant changes of LDPI were observed (Tables 3 and 4).

In SSc patients with PAH, after 4 weeks of therapy with bosentan $62.5 \mathrm{mg}$ bid, the perfusion distribution pattern and the perfusion proximal-distal gradient showed no significant changes from baseline (Table 2). The mean values for minimum, mean, and maximum perfusion and SD did not show significant changes from baseline (Table 3 and Figure 2).

Therefore, in SSc patients with PAH, after 8 and 16 weeks of therapy, the perfusion proximal-distal gradient was seen in a greater number of patients ( 3 patients at 8 weeks vs 11 patients at 16 weeks; $\mathrm{p}<0.05)$, but significant changes were not observed in the perfusion distribution pattern (Table 2). Also, the mean values of minimum, mean, and maximum perfusion and SD showed significant changes from baseline (Table 3 and Figure 2). The mean perfusion was increased significantly in ROI 1 and ROI 2, although it did not show significant changes in ROI 3 (Figure 3 and Table 4).

Major changes from baseline were observed in the early and active capillaroscopic patterns, but small changes were seen in the group of patients with the late pattern (Table 5).

After 8 and 16 weeks of therapy the proximal-distal gradient was present in a significant number of patients $(57 \%)$ with the early capillaroscopic pattern (Table 5).

Table 6 shows LDPI findings in the 3 groups of SSc patients without $\mathrm{PAH}$; no changes from baseline were observed in the 3 capillaroscopic patterns.

Table 2. Laser Doppler perfusion imaging findings in 3 groups of the study population.

\begin{tabular}{llcc}
\hline Timepoint & Group, n (\%) & $\begin{array}{c}\text { Presence of Homogeneous } \\
\text { Perfusion Pattern } \\
\text { n (\%) }\end{array}$ & $\begin{array}{c}\text { Presence of Perfusion } \\
\text { Proximal-Distal Gradient } \\
\text { n (\%) }\end{array}$ \\
\hline \multirow{2}{*}{ Baseline } & SSc patients with PAH & $2(7)$ & $3(10)$ \\
& SSc patients without PAH & $3(10)$ & $2(7)$ \\
& Healthy controls & $29(97)$ & $27(90)$ \\
\multirow{5}{*}{8 weeks weeks } & SSc patients with PAH & $3(10)$ & $2(7)$ \\
& SSc patients without PAH & $3(10)$ & $2(7)$ \\
& Healthy controls & $29(97)$ & $28(93)$ \\
& SSc patients with PAH & $2(7)$ & $11(36)^{*}$ \\
& SSc patients without PAH & $2(7)$ & $3(10)$ \\
& Healthy controls & $29(97)$ & $28(93)$ \\
& SSc patients with PAH & $2(7)$ & $11(36)^{*}$ \\
& SSc patients without PAH & $3(10)$ & $3(10)$ \\
& Healthy controls & $29(97)$ & $28(93)$ \\
\hline
\end{tabular}

*p $<0.05$ compared with baseline. 
Table 3. Laser Doppler perfusion imaging findings in 3 groups of the study population. Mean ( \pm SD) values of minimum, mean, and maximum perfusion are expressed as perfusion units.

\begin{tabular}{|c|c|c|c|c|c|}
\hline Timepoint & Group & Minimum Perfusion & Mean Perfusion & Maximum Perfusion & SD Mean Value \\
\hline \multirow[t]{2}{*}{ Baseline } & SSc patients with PAH & $0.164 \pm 0.070$ & $0.795 \pm 0.119$ & $2.363 \pm 0.429$ & $0.292 \pm 0.050$ \\
\hline & SSc patients without PAH & $0.169 \pm 0.076$ & $0.784 \pm 0.144$ & $2.296 \pm 0.523$ & $0.282 \pm 0.048$ \\
\hline \multirow[t]{3}{*}{4 weeks } & SSc patients with $\mathrm{PAH}$ & $0.170 \pm 0.066$ & $0.800 \pm 0.122$ & $2.302 \pm 0.434$ & $0.291 \pm 0.047$ \\
\hline & SSc patients without PAH & $0.166 \pm 0.072$ & $0.781 \pm 0.148$ & $2.289 \pm 0.518$ & $0.278 \pm 0.043$ \\
\hline & Healthy controls & $0.190 \pm 0.064$ & $0.848 \pm 0.192$ & $2.744 \pm 0.7623$ & $0.311 \pm 0.044$ \\
\hline 8 weeks & Healthy controls & $0.194 \pm 0.063$ & $0.858 \pm 0.177$ & $2.812 \pm 0.786$ & $0.307 \pm 0.048$ \\
\hline \multirow[t]{3}{*}{16 weeks } & SSc patients with PAH & $0.210 \pm 0.078^{*}$ & $0.915 \pm 0.178 * *$ & $2.911 \pm 0.522 *$ & $0.333 \pm 0.049 * * *$ \\
\hline & SSc patients without PAH & $0.170 \pm 0.076$ & $0.790 \pm 0.144$ & $2.290 \pm 0.493$ & $0.284 \pm 0.052$ \\
\hline & Healthy controls & $0.196 \pm 0.059$ & $0.860 \pm 0.167$ & $2.810 \pm 0.746$ & $0.310 \pm 0.050$ \\
\hline
\end{tabular}

$* \mathrm{p}<0.05, * * \mathrm{p}<0.01$, and $\mathrm{p}<0.001$ compared with baseline.

Table 4. Mean $( \pm \mathrm{SD})$ values, expressed as perfusion units of mean perfusion in 3 regions of interest (ROI).

\begin{tabular}{llccc}
\hline Timepoint & Group & Mean Perfusion ROI 1 & Mean Perfusion ROI 2 & Mean Perfusion ROI 3 \\
\hline Baseline & SSc patients with PAH & $0.844 \pm 0.233$ & $0.802 \pm 0.178$ & $0.822 \pm 0.189$ \\
& SSc patients without PAH & $0.788 \pm 0.143$ & $0.796 \pm 0.143$ & $0.787 \pm 0.151$ \\
& Healthy controls & $1.542 \pm 0.238$ & $0.895 \pm 0.197$ & $0.842 \pm 0.171$ \\
4 weeks & SSc patients with PAH & $0.847 \pm 0.275$ & $0.812 \pm 0.180$ & $0.832 \pm 0.180$ \\
& SSc patients without PAH & $0.786 \pm 0.140$ & $0.798 \pm 0.141$ & $0.785 \pm 0.147$ \\
& Healthy controls & $1.553 \pm 0.228$ & $0.894 \pm 0.190$ & $0.844 \pm 0.175$ \\
& SSc patients with PAH & $1.533 \pm 0.244^{*}$ & $1.141 \pm 0.334^{*}$ & $0.866 \pm 0.222 \mathrm{NS}$ \\
& SSc patients without PAH & $0.794 \pm 0.154$ & $0.776 \pm 0.158$ & $0.794 \pm 0.140$ \\
& Healthy controls & $1.561 \pm 0.234$ & $0.845 \pm 0.188$ & $0.822 \pm 0.182$ \\
& SSc patients with PAH & $1.536 \pm 0.254^{*}$ & $1.140 \pm 0.324^{*}$ & $0.846 \pm 0.236 \mathrm{NS}$ \\
& SSc patients without PAH & $0.784 \pm 0.164$ & $0.794 \pm 0.148$ & $0.790 \pm 0.138$ \\
& Healthy controls & $1.566 \pm 0.248$ & $0.888 \pm 0.198$ & $0.848 \pm 0.192$ \\
\hline
\end{tabular}

$* \mathrm{p}<0.01$ compared with baseline; NS: no significant change from baseline.

Table 5. Laser Doppler perfusion imaging measures in 3 capillaroscopic patterns in SSc patients with pulmonary arterial hypertension: early $(\mathrm{n}=14)$, active $(n=9)$, and late $(n=7)$.

\begin{tabular}{|c|c|c|c|c|c|c|c|}
\hline Timepoint & Group & $\begin{array}{l}\text { Minimum } \\
\text { Perfusion, } \\
\text { mean } \pm \text { SD }\end{array}$ & $\begin{array}{c}\text { Mean } \\
\text { Perfusion, } \\
\text { mean } \pm \text { SD }\end{array}$ & $\begin{array}{l}\text { Maximum } \\
\text { Perfusion, } \\
\text { mean } \pm \text { SD }\end{array}$ & $\begin{array}{l}\text { SD Mean Value } \\
\text { Mean } \pm \text { SD }\end{array}$ & $\begin{array}{l}\text { Presence of } \\
\text { Homogeneous } \\
\text { Perfusion } \\
\text { Pattern }\end{array}$ & $\begin{array}{c}\text { Presence of } \\
\text { Perfusion } \\
\text { Proximal-Distal } \\
\text { Gradient }\end{array}$ \\
\hline \multirow[t]{3}{*}{ Baseline } & Early & $0.190 \pm 0.063$ & $0.826 \pm 0.102$ & $2.345 \pm 0.300$ & $0.272 \pm 0.065$ & 0 & $2(14)$ \\
\hline & Active & $0.195 \pm 0.075$ & $0.881 \pm 0.136$ & $2.640 \pm 0.450$ & $0.298 \pm 0.056$ & $1(11)$ & $1(11)$ \\
\hline & Late & $0.121 \pm 0.051$ & $0.750 \pm 0.084$ & $1.978 \pm 0.395$ & $0.221 \pm 0.035$ & $1(14)$ & 0 \\
\hline 4 weeks & Late & $0.123 \pm 0.052$ & $0.749 \pm 0.086$ & $2.077 \pm 0.422$ & $0.224 \pm 0.036$ & $1(14)$ & 0 \\
\hline \multirow{3}{*}{8 weeks } & Early & $0.272 \pm 0.087 *$ & $0.997 \pm 0.185^{*}$ & $2.702 \pm 0.396^{*}$ & $0.317 \pm 0.066^{*}$ & 0 & $8(57)^{*}$ \\
\hline & Active & $0.225 \pm 0.0626^{*}$ & $0.990 \pm 0.128^{*}$ & $3.478 \pm 0.664 * * *$ & $0.326 \pm 0.035^{* *}$ & $1(11)$ & $3(33)$ \\
\hline & Late & $0.138 \pm 0.055 \mathrm{NS}$ & $0.757 \pm 0.046 \mathrm{NS}$ & $2.098 \pm 0.512 \mathrm{NS}$ & $0.232 \pm 0.056 \mathrm{NS}$ & $1(14)$ & 0 \\
\hline \multirow[t]{2}{*}{16 weeks } & Early & $0.278 \pm 0.088^{*}$ & $0.995 \pm 0.188^{*}$ & $2.716 \pm 0.386^{*}$ & $0.316 \pm 0.056^{*}$ & 0 & $8(57)^{*}$ \\
\hline & Active & $0.228 \pm 0.0612^{*}$ & $0.990 \pm 0.130^{*}$ & $3.470 \pm 0.668 * * *$ & $0.326 \pm 0.038^{* *}$ & $1(11)$ & $3(33)$ \\
\hline
\end{tabular}

$* \mathrm{p}<0.05, * * \mathrm{p}<0.01$ and $* * * \mathrm{p}<0.001$ compared with baseline. NS: not significant. 

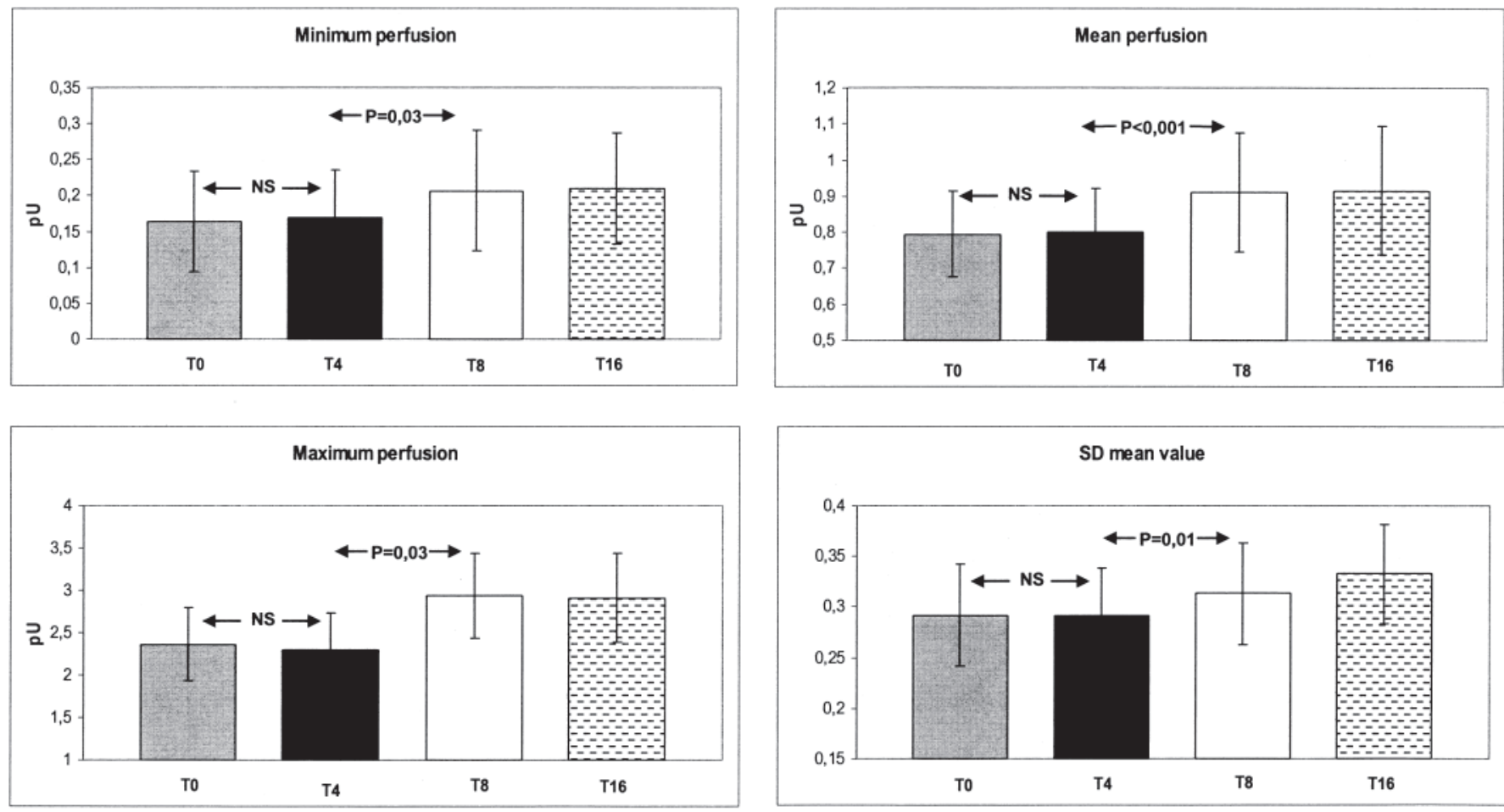

Figure 2. Variations of measures of laser Doppler perfusion imaging in SSc patients with pulmonary artery hypertension at baseline (T0) and after 4, 8, and 16 weeks. Mean values for minimum, mean, and maximum perfusion and SD did not show significant changes from baseline. pU: arbitrary perfusion units; NS: nonsignificant.

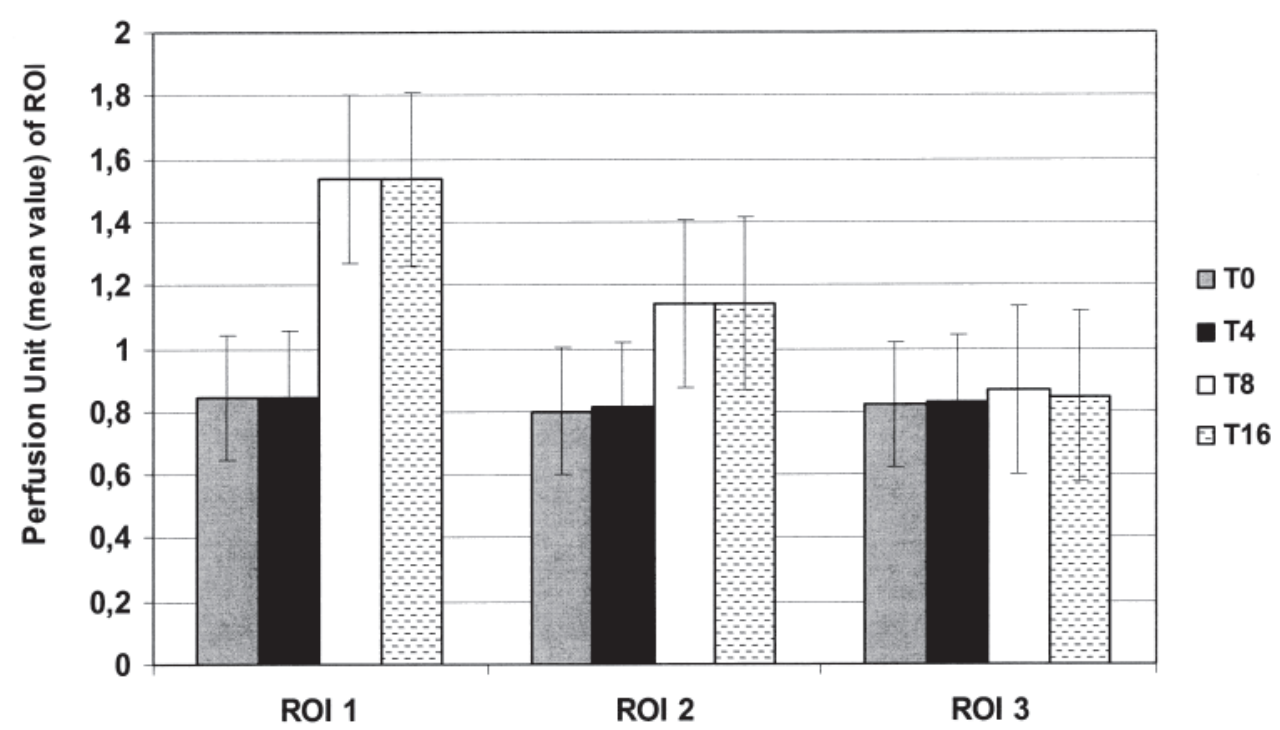

Figure 3. Mean perfusion of 3 regions of interest (ROI) in SSc patients with pulmonary artery hypertension at baseline (T0) and after 4, 8, and 16 weeks. Mean perfusion was increased significantly in ROI 1 and ROI 2, and did not show significant changes in ROI 3.

No significant differences were observed in the groups of patients with limited or diffuse SSc. No significant differences of hand perfusion were observed in patients with and without history of digital ulcers.

Raynaud's Condition Score. We observed no significant differences in RCS in SSc patients with or without PAH. In patients with PAH the RCS was $4.5 \pm 2.0$ at baseline, $4.3 \pm$ 1.9 at 4 weeks, $4.4 \pm 2.1$ at 8 weeks, and $4.4 \pm 1.9$ at 16 weeks. In patients without PAH the RCS was $4.7 \pm 2.6$ at baseline, $4.5 \pm 2.0$ at 4 weeks, $4.4 \pm 2.3$ at 8 weeks, and 4.5 \pm 2.4 at 16 weeks.

The RCS showed no significant differences even in the 
Table 6. Laser Doppler perfusion imaging measures in 3 capillaroscopic patterns in SSc patients without pulmonary arterial hypertension.

\begin{tabular}{|c|c|c|c|c|c|c|c|}
\hline Timepoint & Group & $\begin{array}{l}\text { Minimum } \\
\text { Perfusion, } \\
\text { mean } \pm \text { SD }\end{array}$ & $\begin{array}{c}\text { Mean } \\
\text { Perfusion, } \\
\text { mean } \pm \text { SD }\end{array}$ & $\begin{array}{l}\text { Maximum } \\
\text { Perfusion, } \\
\text { mean } \pm \text { SD }\end{array}$ & $\begin{array}{l}\text { SD Mean Value } \\
\text { Mean } \pm \text { SD }\end{array}$ & $\begin{array}{l}\text { Presence of } \\
\text { Homogeneous } \\
\text { Perfusion } \\
\text { Pattern, n (\%) }\end{array}$ & $\begin{array}{c}\text { Presence of } \\
\text { Perfusion } \\
\text { Proximal-Distal } \\
\text { Gradient, n (\%) }\end{array}$ \\
\hline \multirow[t]{3}{*}{ Baseline } & Early $(\mathrm{n}=14)$ & $0.188 \pm 0.057$ & $0.81 \pm 0.101$ & $2.392 \pm 0.287$ & $0.276 \pm 0.055$ & $1(11)$ & $1(11)$ \\
\hline & Active $(\mathrm{n}=9)$ & $0.195 \pm 0.067$ & $0.873 \pm 0.136$ & $2.648 \pm 0.411$ & $0.301 \pm 0.051$ & $2(14)$ & $1(11)$ \\
\hline & Late $(\mathrm{n}=7)$ & $0.112 \pm 0.038$ & $0.71 \pm 0.072$ & $1.995 \pm 0.377$ & $0.222 \pm 0.027$ & 0 & 0 \\
\hline
\end{tabular}

groups of patients with limited or diffuse SSc, or in the groups with different capillaroscopic patterns.

Nailfold videocapillaroscopy and other outcomes. Bosentan treatment did not result in any significant changes in microvascular architecture and total number of capillary loops. We observed no changes in the Medsger Disease Severity Scale, the Valentini Disease Activity Index, or the modified Rodnan skin score.

Safety. Serum transaminase levels were found to be elevated to $<3$-fold the upper limit of normal in 3 patients treated with bosentan. This increase was transient and did not require dose adjustment.

\section{DISCUSSION}

The typical hallmark of SSc is microvascular involvement; macrovascular involvement is not well documented in these patients, but the majority of investigators agree that its prevalence is similar to that in general population. Early disease is mediated through microvascular dysfunction secondary to a number of factors including endothelial damage, overexpression of specific adhesion molecules, and perivascular inflammatory cell infiltration ${ }^{16,17}$. In this process, endothelial cell injury, fixed structural microvascular changes, and tissue fibrosis play a key role. Endothelial injury also leads to increased release of ET-1, a 21-amino acid peptide produced by endothelial cells. Increased ET-1 levels are found in the serum of patients with SSc, suggesting that it plays a role in the pathogenesis of SSc vascular disease $^{18}$. The beneficial effect of bosentan in PAH and in preventing digital ulcers suggests that the drug promotes vascular function in diverse organ systems ${ }^{7}$.

Recently, LDPI has been used for evaluating skin microcirculation. Rosato, et al demonstrated that LDPI is an accurate method for evaluating skin blood flow in patients with $\mathrm{RP}$ primary or secondary to $\mathrm{SSc}^{9}$. Other instrumental procedures have been used recently to assess cutaneous blood flow. In a recent study Mugii, et $a l^{19}$ demonstrated that reduced red blood cell velocity in nailfold capillaries is a sensitive and specific indicator of microcirculation injury in SSc. The mean red blood cell velocity was significantly decreased in patients with SSc compared to healthy controls and particularly in patients with the active and late NVC pattern. Measurement of red blood cell velocity may be useful in evaluating therapeutic effects on microcirculation ${ }^{19}$.
Cutolo, et al have also demonstrated that fingertip blood perfusion, assessed by laser Doppler flowmetry, is significantly lower in patients with SSc compared to controls. Patients with SSc with the late NVC pattern of microangiopathy had significantly lower fingertip blood perfusion than patients with the active and early NVC patterns. In SSc patients iloprost treatment increased fingertip blood perfusion ${ }^{20}$.

In our study, bosentan improved the LDPI measures (minimum, mean, and maximum perfusion and SD) after 8 and 16 weeks of treatment in SSc patients with PAH. After 4 weeks of oral therapy with bosentan $62.5 \mathrm{mg}$ bid, the LDPI measures showed no significant differences from baseline. Conversely, after 8 and 16 weeks of bosentan therapy, the LDPI values showed significant changes from baseline, and these changes were more evident in patients with the early/active capillaroscopic pattern than in patients with the late pattern. After 4 weeks of therapy the lack of significant differences from baseline upon LDPI evaluation could be due to the lower dose and/or shorter duration of bosentan treatment.

After 8 and 16 weeks of bosentan therapy the skin blood flow improvement was more strongly represented in the ROI 1 than in ROI 2 and ROI 3. In 57\% of the SSc patients with the early capillaroscopic pattern, bosentan restored the proximal-distal perfusion gradient. Although we found no significant differences of LDPI values in patients with and without a history of digital ulcers, the increased skin blood perfusion in ROI 1 could have a relationship with the documented effect of bosentan in the prevention of digital ulcers. Indeed, in the RAPIDS-1 study only ulcers at or distal to the proximal interphalangeal joint were scored ${ }^{7}$. Future studies are needed to determine whether LDPI findings in SSc patients with active digital ulcers are different from those of patients without active digital ulcers.

Although digital ulcers occur on the palmar surface of fingers, we studied the dorsum of hands to remove the artefacts of tremor. However, in our experience, after removal of artefacts the LDPI images of the dorsum are comparable to those of the palm of hands.

Other studies, although conducted with different instrumental methods, have shown that ET-1 receptor antagonists improve peripheral vascular reactivity. A recent study showed that nonselective ETA/B blockade by systemic 
administration of bosentan was associated with improved flow-mediated, nitric oxide-dependent vasodilation in patients with $\mathrm{SSc}^{21}$. Cardillo, et $a l^{22}$ demonstrated that endothelium-dependent vasodilator responsiveness to acetylcholine in patients with SSc was reversed after blockade of ETA receptors. In the absence of ETA antagonism, patients exhibited impaired vasodilator response to acetylcholine, whereas the endothelium-independent vasorelaxation effect of sodium nitroprusside was not different in the SSc patients compared with healthy controls ${ }^{22}$. In another study, treatment with bosentan after 16 weeks appeared to improve peripheral thermoregulation evaluated by thermography in patients with secondary RP, independent of digital ulcers $^{23}$. Dunne, et al demonstrated in patients with SSc that blood flow measured by laser Doppler fluximetry improved after bosentan treatment ${ }^{24}$. Conversely, in a small group of patients with limited SSc, bosentan did not result in any significant change in microvascular endothelial-dependent and endothelial-independent vasodilation evaluated by laser Doppler fluximetry in combination with iontophoresis 25 .

Endothelial-dependent vasodilatation is very important in the regulation of microcirculatory flow. In SSc, abnormal endothelial vasodilator function seems to be the early stage of the process that adversely influences the microvascular bed, with a reduction in the size of microvessels leading to decreased blood flow to organs and, ultimately, to a state of chronic ischemia. Because activation of the ET-1 system also plays a role in determining endothelial dysfunction in $\mathrm{SSc}^{26,27,28}$, we can suppose that bosentan ameliorates endothelial dysfunction and consequently microcirculatory flow.

In previous studies, bosentan did not result in any significant improvement in $\mathrm{RCS}^{29}$. A recent study showed that in the absence of ETA antagonism SSc patients exhibited impaired vasodilator response to acetylcholine (endothelium-dependent vasorelaxation), whereas the endotheliumindependent vasorelaxation effect of sodium nitroprusside was not different in SSc patients compared with healthy controls $^{22}$. Others showed that blood flow determined by photoelectric plethysmography during cooling and rewarming did not improve during treatment with bosentan ${ }^{30}$. Therefore we can assume that ET-1 receptor antagonism improves endothelium-dependent vasorelaxation, but it does not exert any action on endothelium-independent vasorelaxation factors (e.g., physical factors like temperature and pressure, the autonomous nervous system).

Thus, our study is restricted by its limitations: we omitted correction for temperature, the open-label approach, the lack of a run-in treatment-free period, the lack of a crossover design. Since PAH is a diagnosis of exclusion, the correct diagnostic algorithm includes right heart catheterization (RHC). Transthoracic echocardiography provides several variables that correlate with RHC, including PAP, and should always be performed in cases of suspected PAH.
Unfortunately, despite the strong correlation of the tricuspid regurgitation velocity and tricuspid regurgitation pressure gradient, Doppler-derived pressure estimation may be inaccurate in the individual patient. RHC is required to confirm the diagnosis of PAH, to assess the severity of the hemodynamic impairment, and to test the vasoreactivity of the pulmonary circulation. Since our objective was to evaluate the effects of bosentan on skin perfusion, errors in assessing the PAH linked to the lack of RHC did not influence the outcomes of the study.

In summary, bosentan improved skin perfusion in SSc patients with PAH, although it did not ameliorate symptoms of RP. In SSc patients with PAH, skin blood perfusion increased, particularly in skin regions distal to the proximal interphalangeal joint and in patients with the early/active capillaroscopic pattern. Future double-blinded randomized clinical trials are needed to evaluate the effects of bosentan on skin perfusion assessed in SSc patients without PAH and with active digital ulcers.

\section{REFERENCES}

1. LeRoy EC, Black C, Fleischmajer R, Jablonska S, Krieg T, Medsger TA Jr, et al. Scleroderma (systemic sclerosis): classification, subsets and pathogenesis. J Rheumatol 1998;15:202-5.

2. Kahaleh MB. Vascular involvement in systemic sclerosis (SSc). Clin Exp Rheumatol 2004;22 Suppl 33:19-23.

3. Herrick AL. Pathogenesis of Raynaud's phenomenon. Rheumatology 2005;44:587-96.

4. Flavahan NA, Flavahan S, Mitra S, Chotani MA. The vasculopathy of Raynaud's phenomenon and scleroderma. Rheum Dis Clin North Am 2003;29:275-91.

5. Salsano F, Letizia C, Proietti M, Rossi C, Proietti AR, Rosato E, et al. Significant changes of peripheral perfusion and plasma adrenomedullin levels in acetylcysteine long term treatment of patients with sclerodermic Raynaud's phenomenon. Int J Immunopathol Pharmacol 2005;18:761-70.

6. Denton CP, Humbert M, Rubin L, Black CM. Bosentan treatment for pulmonary arterial hypertension related to connective tissue disease: a subgroup analysis of the pivotal clinical trials and their open-label extensions. Ann Rheum Dis 2006;65:1336-40.

7. Korn JH, Mayes M, Matucci Cerinic M, Rainisio M, Pope J, Hachulla E, et al. Digital ulcers in systemic sclerosis: prevention by treatment with bosentan, an oral endothelin receptor antagonist. Arthritis Rheum 2004;50:3985-93.

8. Murray AK, Moore TL, Manning JB, Taylor C, Griffiths CE, Herrick AL. Noninvasive imaging techniques in the assessment of scleroderma spectrum disorders. Arthritis Rheum 2009;61:1103-11.

9. Rosato E, Borghese F, Pisarri S, Salsano F. Laser Doppler perfusion imaging is useful in the study of Raynaud's phenomenon and improves the capillaroscopic diagnosis. J Rheumatol 2009;36:2257-63.

10. Preliminary criteria for the classification of systemic sclerosis (scleroderma). Subcommittee for scleroderma criteria of the American Rheumatism Association Diagnostic and Therapeutic Criteria Committee. Arthritis Rheum 1980;23:581-90.

11. Rubin LJ; American College of Chest Physicians. Diagnosis and management of pulmonary arterial hypertension: ACCP evidence-based clinical practice guidelines. Chest 2004;126 Suppl 1:7-10.

12. Valentini G, Silman AJ, Veale D. Assessment of disease activity. 
Clin Exp Rheumatol 2003;21 Suppl 29:39-41.

13. Medsger TA Jr, Bombardieri S, Czirjak L, Scorza R, Della Rossa A, Bencivelli W. Assessment of severity and prognosis. Clin Exp Rheumatol 2003;21 Suppl 29:42-6.

14. Cutolo M, Sulli A, Pizzorni C, Accardo S. Nailfold videocapillaroscopy assessment of microvascular damage in systemic sclerosis. J Rheumatol 2000;27:155-60.

15. Merkel PA, Herlyn K, Martin RW, Anderson JJ, Mayes MD, Bell P, et al. Scleroderma Clinical Trials Consortium. Measuring disease activity and functional status in patients with scleroderma and Raynaud's phenomenon. Arthritis Rheum 2002;46:2410-20.

16. Yamane K. Endothelin and collagen vascular disease: a review with special reference to Raynaud's phenomenon and systemic sclerosis. Intern Med 1994;33:579-82.

17. Herrick AL. Vascular function in systemic sclerosis. Curr Opin Rheumatol 2000;12:527-33.

18. Vancheeswaran R, Magoulas T, Efrat G, Wheeler-Jones C, Olsen I, Penny R, et al. Circulating endothelin-1 levels in systemic sclerosis subsets - a marker of fibrosis or vascular dysfunction? J Rheumatol 1994;21:1838-44.

19. Mugii N, Hasegawa M, Hamaguchi Y, Tanaka C, Kaji K, Komura $\mathrm{K}$, et al. Reduced red blood cell velocity in nail-fold capillaries as a sensitive and specific indicator of microcirculation injury in systemic sclerosis. Rheumatology 2009;48:696-703.

20. Cutolo M, Ferrone C, Pizzorni C, Soldano S, Seriolo B, Sulli A. Peripheral blood perfusion correlates with microvascular abnormalities in systemic sclerosis: A laser-Doppler and nailfold videocapillaroscopy study. J Rheumatol 2010;37:1174-80.

21. Sfikakis PP, Papamichael C, Stamatelopoulos KS, Tousoulis D, Fragiadaki KG, Katsichti P, et al. Improvement of vascular endothelial function using the oral endothelin receptor antagonist bosentan in patients with systemic sclerosis. Arthritis Rheum 2007;56:1985-93.

22. Cardillo C, Schinzari F, Melina D, Mores N, Bosello S, Peluso G, et al. Improved endothelial function after endothelin receptor blockade in patients with systemic sclerosis. Arthritis Rheum 2009;60:1840-4.
23. Selenko-Gebauer N, Duschek N, Minimair G, Stingl G, Karlhofer F. Successful treatment of patients with severe secondary Raynaud's phenomenon with the endothelin receptor antagonist bosentan. Rheumatology 2006;45 Suppl:45-8.

24. Dunne J, Dutz J, Shojania K, Ng B, Van Eeden S. Treatment of severe Raynaud's phenomenon with bosentan in a patient with systemic sclerosis. Rheumatology 2006;45:911-2.

25. Hettema ME, Zhang D, Stienstra Y, Smit AJ, Bootsma H, Kallenberg CG. No effects of bosentan on microvasculature in patients with limited cutaneous systemic sclerosis. Clin Rheumatol 2009;28:825-33.

26. Grunfeld S, Hamilton CA, Mesaros S, McClain SW, Dominiczak $\mathrm{AF}$, Bohr DF, et al. Role of superoxide in the depressed nitric oxide production by the endothelium of genetically hypertensive rats. Hypertension 1995;26:854-7.

27. Taddei S, Virdis A, Ghiadoni L, Magagna A, Salvetti A. Vitamin C improves endothelium-dependent vasodilation by restoring nitric oxide activity in essential hypertension. Circulation 1998;97:2222-9.

28. Shi-wen X, Kennedy L, Renzoni EA, Bou-Gharios G, du Bois RM, Black CM, et al. Endothelin is a downstream mediator of profibrotic responses to transforming growth factor $B$ in human lung fibroblasts. Arthritis Rheum 2007;56:4189-94.

29. Nguyen VA, Eisendle K, Gruber I, Hugl B, Reider D, Reider N, et al. Effect of the dual endothelin receptor antagonist bosentan on Raynaud's phenomenon secondary to systemic sclerosis: a double-blind prospective, randomized, placebo-controlled pilot study. Rheumatology 2010;49:583-7.

30. Hettema ME, Zhang D, Bootsma H, Kallenberg CGM. Bosentan therapy for patients with severe Raynaud's phenomenon in systemic sclerosis. Ann Rheum Dis 2007;66:1398-9. 